\title{
Monitoring insect pest infestation via different spectroscopic techniques
}

\begin{abstract}
ABSTRCT
Nowadays, monitoring and observing insect pest populations is a major and crucial issue in agriculture, especially for crop protection. Spectroscopic techniques are well recognized for detecting and monitoring insect pests in the field and also the internal quality of fruit. There are several spectroscopic techniques with specific characteristic features, including mass spectrometry, ultraviolet-visible spectroscopy, infrared spectroscopy, and nuclear resonance spectroscopy. Nevertheless, limitations and complexity are the constraints of these technologies. In this paper, the spectroscopic and imaging spectroscopic techniques are discussed, compared, and investigated, namely fluorescence light detection and ranging (LIDAR) to study the fluorescence of diverse types of planthopper (Hemiptera) and moth (Lepidoptera), visible and near-infrared (Vis/NIR) spectroscopy to detect internal insect-infested jujubes, NIR spectroscopy to determine spectral properties of oil palm bagworms, hyperspectral transmittance image for detecting insect-damaged vegetable soybeans, and remote sensing measurement to detect bagworm infestation in oil palm plantations. These techniques are found to be reliable methods for better monitoring of insect pest movement in the harvested plant and in the ground, for the detection of insect-damaged vegetable soybeans and internal insect infestation in jujubes, and for the determination of oil palm bagworm spectral properties.
\end{abstract}

Keyword: Insect pest; Visible/near-infrared; NIR; LIDAR ;Hyperspectral. 